\title{
The One Year Fate of Iron Oxide Coated Gold Nanoparticles in Mice
}

\author{
Jelena Kolosnjaj-Tabi, ${ }^{t, \pm,+}$ Yasir Javed, ${ }^{\text {s,+ }}$ Lénaic Lartigue, ${ }^{\dagger, \S}$ Jeanne Volatron, ${ }^{\dagger}$ Dan Elgrabli, ${ }^{\dagger}$ Iris Marangon, ${ }^{\dagger}$ \\ Giammarino Pugliese, ${ }^{\perp}$ Benoit Caron,, Albert Figuerola, ${ }^{\perp}$ Nathalie Luciani, ${ }^{\dagger}$ Teresa Pellegrino, ${ }^{\perp}$ \\ Damien Alloyeau, ${ }^{\S}$ and Florence Gazeau*, ${ }^{*}$ \\ †Laboratoire Matières et Systèmes Complexes, UMR 7057 CNRS/Université Paris Diderot, 10 rue Alice Domon et Léonie Duquet, Paris F-75205 Cedex 13, France, \\ ${ }^{\ddagger}$ Inserm U970, Paris Cardiovascular Research Center-PARCC/Université Paris-Descartes, 56 rue Leblanc, Paris 75015, France, ${ }^{\S}$ Laboratoire Matériaux et Phénomènes \\ Quantiques, UMR 7162 CNRS/Université Paris Diderot, 10 rue Alice Domon et Léonie Duquet, Paris F-75205 Cedex 13, France, "ISTeP, UMR 7193 CNRS/Université \\ Pierre et Marie Curie, 4 place Jussieu, Paris 75005 , France, and ${ }^{\perp}$ Istituto Italiano di Tecnologia, via Morego 30, Genova 16163 , Italy. ${ }^{+}$These authors contributed \\ equally.
}

\begin{abstract}
Safe implementation of nanotechnology and nanomedicine requires an in-depth understanding of the life cycle of nanoparticles in the body. Here, we investigate the long-term fate of gold/iron oxide heterostructures after intravenous injection in mice. We show these heterostructures degrade in vivo and that the magnetic and optical properties change during the degradation process. These particles eventually eliminate from the body. The comparison of two different coating shells for heterostructures, amphiphilic polymer or polyethylene glycol, reveals the long lasting impact of initial surface properties on the nanocrystal degradability and on the kinetics of elimination of magnetic iron and gold from liver and spleen. Modulation of nanoparticles reactivity to the biological environment by the choice of materials and surface functionalization may provide new directions in the design of multifunctional nanomedicines with predictable fate.
\end{abstract}

$\mathrm{T}$ echnological breakthroughs related to innovative nanomaterials in medical and consumer products is accompanied by specific issues regarding the safety and life cycle of nanomaterials in the environment and, more particularly, in the human body. Materials with one or several dimensions smaller than $100 \mathrm{~nm}$ have increasing complex and dynamic interactions with both environmental and biological systems. ${ }^{1,2}$ An overall objective is to correlate the synthetic identity of nanoparticles to their behavior and transformations in living organisms, and their physicochemical properties with biological functions. ${ }^{3-8}$ Understanding the (bio)transformations of nanomaterials over their entire life cycle is thus a pivotal challenge of nanoscience and a critical issue for societal acceptability. ${ }^{9}$

While most nanotoxicology studies focus on the biochemical effects caused by particles, ${ }^{10}$ the information on the transformations inflicted by the biological environment to the nanoparticles are still scarce or limited to a short time frame after administration. ${ }^{11-15}$ We recently proposed that the nanoparticles physical properties evolve over their life cycle (e.g., their magnetic properties) and the ability to probe the evolution would allow for the identification of the length of time (i.e., age) of nanoparticles in the organism. ${ }^{16,17}$ Nanoparticles may undergo morphological and structural transformations such as degradation, dissociation, dissolution, agglomeration. Here, we propose to use a combination of qualitative and quantitative analytical techniques to study and monitor the long-term fate and biodegradation of gold/iron oxide nanoheterostructures (NHs) over one year after parenteral administration in mice. Gold and iron oxide nanoparticles are two of the most studied inorganic nanomaterials due to their unique optical and magnetic properties. ${ }^{18,19}$ These NHs may be used as theranostic agents with imaging and therapeutic applications, such as for MRI imaging and hyperthermia. ${ }^{20,21}$ However, the safety of nanoparticles, as well as their efficacy, depends on how they transform in the organism. ${ }^{22}$ While gold 
nanoparticles are considered as a relatively bioinert material, concerns are raised by their persistent accumulation in the body. ${ }^{23-26}$ Moreover, gold particles become catalytic and highly reactive as their size is decreased to a few nanometers and interestingly some biological effects have been reported on small size gold particles. ${ }^{27,28}$ Therefore, the morphological evolution of persistent gold particles in the body should be evaluated and the size-dependent elimination/ excretion resulting from potential in vivo degradation have to be elucidated. ${ }^{23,29}$ In contrast to gold particles, iron oxide may benefit from iron metabolism and can be processed and assimilated by the organism. ${ }^{30,31} \mathrm{We}$ have shown before that the superparamagnetic properties of intravenously administered iron oxide nanospheres and nanocubes were degraded over a few months after accumulation in the liver and the spleen, while the endogenous form of iron stored in ferritin protein was increased. ${ }^{17,32}$ The loss of magnetic properties of the initial nanomagnets was correlated to the degradation of single iron oxide nanocrystal by acidic medium such as the lysosome. ${ }^{32,33}$ Remarkably, the coating of iron oxide nanoparticles drastically affects the kinetics of particle degradation in situ in acidic medium. ${ }^{16,34}$ For example, the edge of nanocubes dissolves when their surface is coated by a less dense polymer, but the remaining parts of the cube retain their original crystalline structure. ${ }^{32}$ However, it is not clear if the same mechanisms of local degradation and protective effect of the coating may be relevant in vivo. Here, we further tested whether coating of $\mathrm{NHs}$ with an amphiphilic polymer and polyethylene glycol influenced the degradation fate over 6 months following injection.

\section{RESULTS AND DISCUSSION}

The heterostructures used in this study consisted of a gold monocrystalline core of $4.7 \pm 1.0 \mathrm{~nm}$ coated with magnetite or maghemite nanocrystals (Figure 1A) with a mean overall size of $13.4 \pm 3.2 \mathrm{~nm}$. They were synthesized by thermal decomposition method following the procedure of Sun. ${ }^{35}$ This nonhydrolytic synthesis consists in the in situ nucleation of gold seeds on which the iron oxide nanocrystals can selectively grow. High-Resolution TEM (HRTEM) observations reveal that gold and iron oxide structures are mostly found in cube-on-cube epitaxy. This epitaxial growth is energetically favorable because the lattice parameter of the inverse spinel structure of magnetite or maghemite $(0.840$ and $0.835 \mathrm{~nm})$ is almost two times larger than a single FCC gold structure $(0.408 \mathrm{~nm})$. This is illustrated in Figure $1 \mathrm{~B}$ showing a heterostructure oriented along the [001] zone axis. The fast Fourier transformations (FFT) calculated on the iron oxide structure and on the superposed gold/iron oxide structures are identical, because the [004] and [440] reflections of iron oxide are superposed with the [002] and [220] reflections of gold.
We note that $\mathrm{NHs}$ with the vacancy-ordered maghemite structure (tetragonal unit cell with the $P 4_{1} 2_{1} 2$ space group) were also observed. ${ }^{36-38}$

As synthesized hydrophobic surfactant-coated $\mathrm{NHs}$ (surfactants: oleic acid, oleylamine and 1,2-hexadecanediol) were transferred to water by two distinct procedures. The first one is based on the intercalation of an amphiphilic polymer, namely, poly(maleic anhydride alt-1-octadecene) of $30-50 \mathrm{kDa}$, on top of the surfactant coated $\mathrm{NHs}$, forming a micelle-like coating. ${ }^{39,40}$ Colloidal stability and negative charges of the particles were confirmed in water by dynamic light scattering (DLS) and electrophoresis (Supporting Information Figure S1). Alternatively, in a second approach we used a catechol-derived polyethylene glycol ligand (gallol-PEG-OH, $3 \mathrm{kDa}$ ) adapted by a previously developed protocol, ${ }^{41}$ to anchor the iron oxide surface with the gallic acid groups by replacing the surfactant molecules present on the as synthesized $\mathrm{NHs}$. Polyethylene glycol molecules stabilize the heterostructures by steric hindrance (Supporting Information Figure S2). ${ }^{42}$ Two different coatings were selected because of their opposite impact on particle circulation in the blood. While PEG coating is known to enhance circulation time, amphiphilic polymers are negatively charged and are expected to be more rapidly captured by liver macrophages. $\mathrm{NHs}$ were stable in water regardless of the coating and had hydrodynamic diameters of about $60 \mathrm{~nm}$. Polymer-coated NHs were stable in $0.9 \%$ $\mathrm{NaCl}$ and in PBS, while PEG-coated particles tended to aggregate in $0.9 \% \mathrm{NaCl}$ in $1 \mathrm{~h}$ and more slowly in PBS (Supporting Information Figure S3). Both PEG-coated and polymer-coated $\mathrm{NHs}$ tended to aggregate in Dulbecco's Modified Eagle's Medium (DMEM) free of fetal bovine serum (FBS), but they were both stable over $48 \mathrm{~h}$ in DMEM with either $10 \%$ or $50 \%$ FBS, with hydrodynamic diameters of 140 and $117 \mathrm{~nm}$ in 10\% FBS and 91 and $62 \mathrm{~nm}$ in 50\% FBS, respectively (Supporting Information Figures S4 and S5). Importantly, gel electrophoresis and TEM of particles that were magnetically separated from the full media $(0,10 \%$ and $50 \%$ FBS $)$ demonstrate the presence of proteins at the surface of NPs for both types of coating (Supporting Information Figures S6 and S7). Therefore, we can conclude that both types of particles interact with proteins when FBS is present in the medium and the protein adsorption (the so-called protein corona) has a stabilizing effect on small $\mathrm{NH}$ agglomerates. The fact that PEG-coated $\mathrm{NHs}$ are less stable in saline solution and unexpectedly interact with proteins may be due to the presence of exposed gold surface: gallic acid has lower affinity toward gold, while in contrast, the gallol group represents a strong ligand moiety for iron oxide surface. On the other hand, the amphiphilic polymer can wrap around the entire $\mathrm{NH}$ both the gold and the iron oxide domains. In support of this point, by thermogravimetric analysis (TGA), a weight loss 

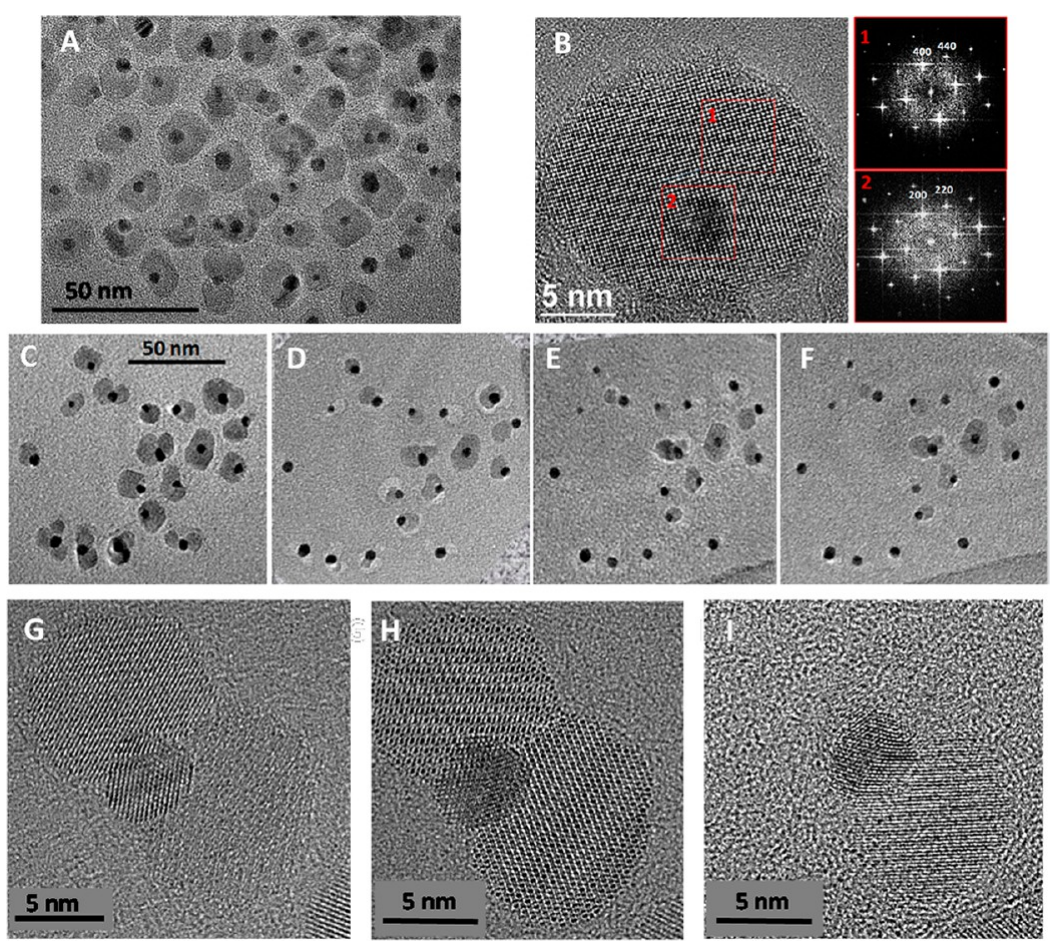

Figure 1. Representative TEM micrographs of the polymer-coated gold/iron heterostructures. (A and B) HRTEM micrograph showing the cube-on-cube epitaxy of gold and iron oxide structures oriented along the [001] zone axis. The FFT in inset 1 was calculated on the iron oxide structure (area 1) and it is indexed with the inverse spinel structure of iron oxide. The FFT in inset 2 was calculated on the superposed iron oxide/gold structures (area 2 ) and it is indexed with the FCC structure of gold. Gold particles are also distinguished by their higher TEM contrast. (C-F) TEM monitoring of single polymer-coated NHs immersed in the acidic medium for different time-points, $0 \mathrm{~h}(\mathrm{C}), 1 \mathrm{~h}(\mathrm{D}), 3 \mathrm{~h}(\mathrm{E})$, and $4 \mathrm{~h}(\mathrm{~F})$. We observe the progressive dissolution of iron oxide moieties, leaving resilient gold particles. Note that the dissolution is not homogeneous among NHs. (G-I) HRTEM of a single $\mathrm{NH}$ degradation after 0,30 , and $60 \mathrm{~min}$ in the lysosome like medium. Iron oxide crystals are organized as petals around the resilient gold core and are eroded with distinctive kinetics.

TABLE 1. Elemental Analysis of the Nanoheterostructures Samples Used in the Study

\begin{tabular}{|c|c|c|c|c|c|c|c|}
\hline & \multicolumn{2}{|c|}{ stock concentration } & \multirow[b]{3}{*}{ Fe/Au Ratio } & \multicolumn{4}{|c|}{ injected dose } \\
\hline & \multirow{2}{*}{$\begin{array}{l}\mathrm{Fe} \\
\mathrm{g} / \mathrm{L}\end{array}$} & \multirow{2}{*}{$\begin{array}{l}\mathrm{Au} \\
\mathrm{g} / \mathrm{L}\end{array}$} & & \multicolumn{2}{|c|}{$\mathrm{Fe}$} & \multicolumn{2}{|c|}{$\mathrm{Au}$} \\
\hline & & & & $\mu \mathrm{mol} / \mathrm{kg}$ & $\mu \mathbf{g}$ & $\mu \mathrm{mol} / \mathrm{kg}$ & $\mu \mathbf{g}$ \\
\hline PC-NHs (polymer-coated) & 6.38 & 1.99 & 3.2 & 50 & 56 & 4.4 & 17.5 \\
\hline PC- hollow NHs & 5.86 & 0.6 & 9.8 & \multicolumn{4}{|c|}{ not injected } \\
\hline PEG-NHs (PEG-coaed) & 4.18 & 0.89 & 4.7 & 50 & 56 & 3.05 & 12 \\
\hline
\end{tabular}

of about $9 \%$ was measured on PEG-coated $\mathrm{NH}$ against a higher loss of $23 \%$ for the PC-NH (see Supporting Information Figure S8). This corresponds to approximately 157 PEG molecules (molecular weight of $3125 \mathrm{~g} / \mathrm{mol}$ ) per $\mathrm{NH}$ and 61 of much longer polymer $(30000 \mathrm{~g} / \mathrm{mol})$ for the PC-NH. Given the difference in ligand number and the factor of 10 on their molecular weight, there is likely a higher surface coverage of the amphiphilic polymer on NHs than PEG.

In addition, it is likely that different types of proteins might adsorb on the particles depending on the ligand charge (amphiphilic polymer provides a more negative surface to the $\mathrm{NH}$ than the neutral PEG) and also on the percentage of serum used in the experiment. ${ }^{43}$ Importantly, both types of particles were injected intravenously in a saline suspension immediately after dilution (within $1 \mathrm{~h}$, the particles show similar stability in $0.9 \% \mathrm{NaCl}$ solution). Gold and iron content in PC- and PEG-coated NHs are summarized in Table 1.

Degradation of NHs in Acidic Medium. In vitro experiments allow monitoring of the morphological transformations of single $\mathrm{NHs}$ in a medium mimicking intracellular lysosomal environment. Particles were immersed in an acidic citrate medium ( $\mathrm{pH}$ 4.7, $20 \mathrm{mM}$ citrate). ${ }^{44}$ Of note, this medium does not fully represent lysosomes, which have different types of enzymes and small molecules in these organelles, but this media will allow us to determine two important parameters $\mathrm{pH}$ and iron chelators) on the transformation of the $\mathrm{NHs}$. Water-suspended polymer-coated NHs were deposited 

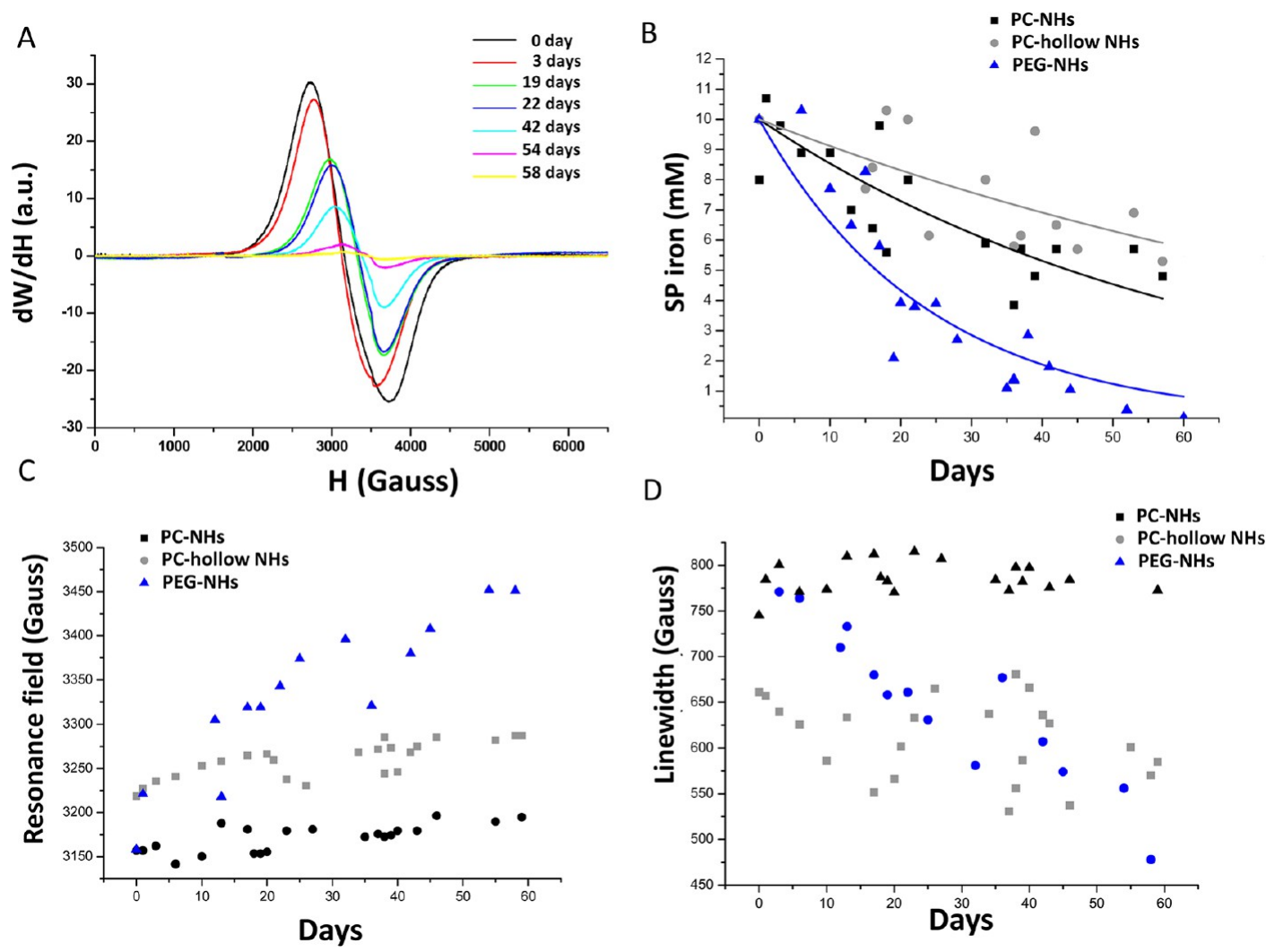

Figure 2. ESR monitoring of superparamagnetic iron from $\mathrm{NHs}$ in acidic citrate medium. Superparamagnetic heterostructures show a characteristic ESR spectrum displayed in (A) for PEG-NHs at different time-points in degradation medium (Initial spectrum of PC-NHs is similar). The measurement of ESR absorption signal provides a specific quantification of superparamagnetic iron (SP iron) represented in (B) as a function of time in degradation medium. Solid lines represent monoexponential fit with a characteristic decay time. Dissolution is faster for PEG-coated NHs (PEG-NHs, decay time of 24 days) in comparison to the polymer-coated hollow (PC-hollow NHs, 108 days) and full NHs (PC-NHs, 63 days). Upon time in degradation medium, we observe a shift of the resonance field toward higher fields (C) and a diminution of the line width (D), which are consistent with the diminution of magnetic volume of the NHs over time. These evolutions are less pronounced for PC-NHs and PC-hollow NHs in comparison to the fast degrading PEG-NHs.

on a lacey-carbon-film-coated copper TEM grid, and their initial morphology was observed. The grid was subsequently immersed in the acidic citrate medium, and the same nanoparticles were observed repeatedly at different time-points. As shown in Figure $1 \mathrm{C}-\mathrm{F}$, the gradual dissolution of iron oxide nanocrystals around unaffected gold cores was observed in a few hours. Such dissolution was not observed in water and physiological medium. As previously described for iron oxide nanocubes, ${ }^{32}$ the dissolution rate is uneven among nanocomposites: some of them are left intact after $4 \mathrm{~h}$ of exposure in the acidic citrate medium, whereas others lost their iron oxide component after 1 and $3 \mathrm{~h}$. Such behavior can be related to the heterogeneity of polymer coverage, which raises a first shield to the access of citrate to the crystal and delays iron oxide dissolution (Supporting Information Figure S9). Importantly, the distinct behavior of gold and iron oxide observed at the nanoscale illustrates the different reactivity of these materials to acidic citrate medium, which favors iron oxide dissolution. Note that the persistent gold seeds pinpoint the initial location of heterostructures and reveal the progressive erosion of iron oxide. The presence of degraded iron oxide crystals around the gold "tracers" rules out the possibility of the detachments of the iron oxide component in the NHs. Furthermore, atomic scale imaging, exemplified in Figure 1G-I, shows that iron oxide conserves its atomic structure after the degradation and that iron oxide/gold interface remains intact. The figure also reveals the lobulated structure of iron oxide around the gold particle. Each lobe or petal ( 1 to 3 per $\mathrm{NH}$ ) having its proper crystalline orientation also degrades independently with its own kinetics. Similar behavior was observed for the PC- (Figure 1) and PEG-coated $\mathrm{NHs}$ (Supporting Information Figure S10), although the iron oxide degradation seemed faster for the PEG-NHs. To investigate if the heterostructure of the particles could play a role on iron oxide degradation, the gold moieties of polymer-coated NHs were dissolved by selective etching of the gold domain using the iodine tincture (Supporting Information Figure S11). ${ }^{45}$ Interestingly the lobulated structure of iron oxide was still observed after gold removal. Iron oxide dissolution in the acidic medium opened a gap between each lobe and tended to separate them (Supporting Information 

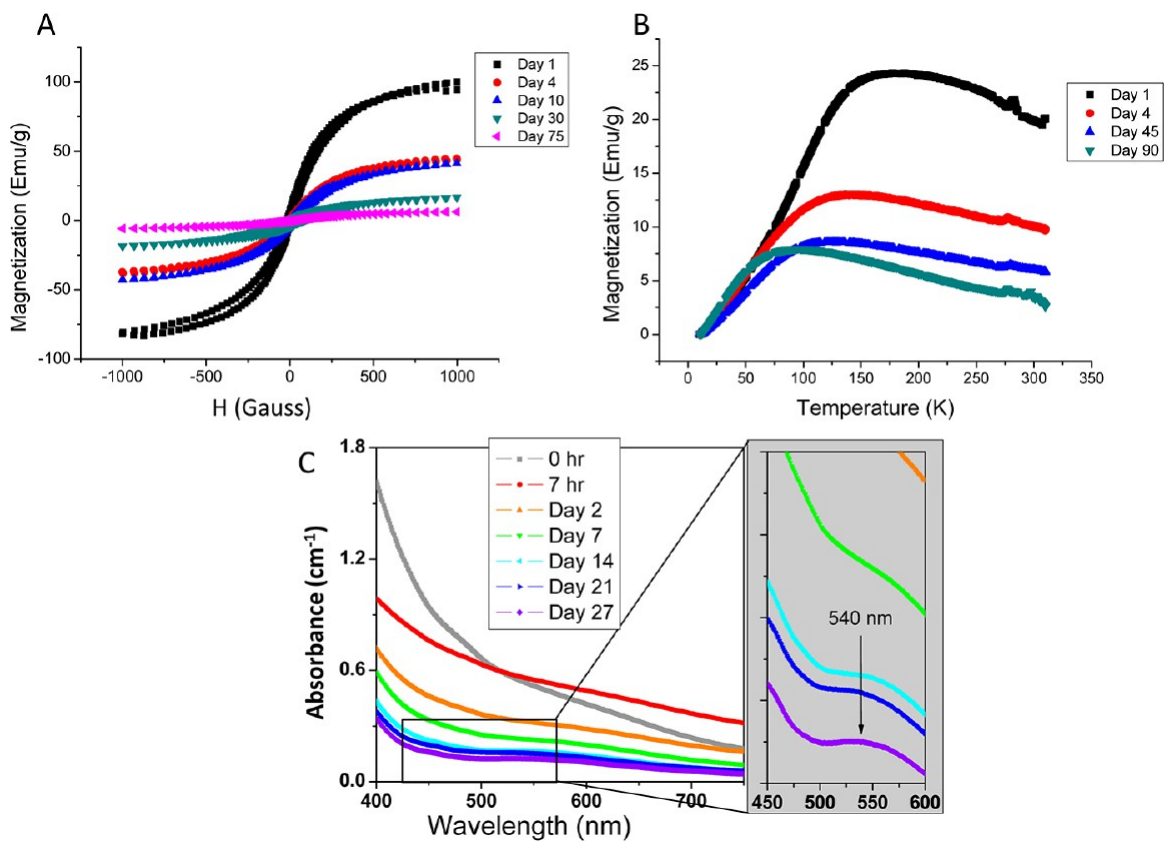

Figure 3. Evolution of the magnetic and optical properties of PEG-coated NHs dispersed in acidic medium. (A) Magnetization as a function of the magnetic field applied. Note the decrease of initial magnetic susceptibility with time in the lysosome-like medium. (B) Magnetization as a function of temperature for zero-field-cooled suspension of NHs. The magnetic field applied is $50 \mathrm{Oe}$. Again the magnetization is decreased upon time, and the blocking temperature of the particles (temperature of maximum magnetization) is shifted toward lower temperatures with time. This shift indicates a reduction of the Néel relaxation time or anisotropy energy of the particles. Overall, the magnetic size of the particles is reduced upon degradation. (C) Absorbance of $\mathrm{NHs}$ is diminishing with time in the acidic citrate medium. Low wavelength extinction due to iron oxide is decreasing, while the plasmon resonance peak of gold particles (see the inset) becomes increasingly more visible with time. Note that the peak (around $540 \mathrm{~nm}$ ) is slightly red-shifted in comparison to that of $5 \mathrm{~nm}$ gold particles $(520 \mathrm{~nm})$ due to the local dielectric environment.

Figure S11). Since the amphiphilic polymer was not affected by the iodine treatment, it might have filled the cavity left by gold dissolution, thus protecting the particles from internal degradation. From HRTEM (Supporting Information Figure S12), we found that the dissolution mainly occurred at the interface of the iron oxide "petals", but that some cavities also became larger due to the degradation of the iron oxide, which suggests the uneven distribution of polymer in the cavity after gold removal. Overall, in situ observations of $\mathrm{NHs}$ degradation confirm the protective roles of polymer and of the gold/iron oxide interface on degradation, shielding the iron oxide from citrate iron chelators. $^{32,33}$

Properties of NHs during Transformation. We next evaluated the evolution of magnetic and optical properties of $\mathrm{NHs}$ when dispersed in the acidic citrate medium at a fixed concentration (10 mM of iron). Electron Spin Resonance (ESR) was used to quantify the amount of iron that contributes to the superparamagnetic properties of the particles (denoted here as superparamagnetic iron). ${ }^{16}$ Original NHs in water suspension display a characteristic ESR spectrum whose total absorption signal is proportional to superparamagnetic iron (Figure 2A). In contrast, free iron ions that can be released from the particles have no ESR signal at room temperature. In acidic citrate medium, the ESR absorption of $\mathrm{NHs}$ decreased in intensity reflecting the loss of superparamagnetic iron over time (Figure 2A,B). Concomitantly ESR resonance field increases while the line width decreases indicating a diminution of magnetic size (Figure 2C,D) ${ }^{46}$ Importantly, as previously observed for iron oxide nanocubes, ${ }^{32}$ PEG-NHs degrade much faster than their amphiphilic polymercoated counterparts. In contrast, removal of the gold core on PC-coated particles does not accelerate the degradation of iron oxide, suggesting a redistribution of the polymer shield. In line with ESR quantification, we observe a drastic decrease of the field-dependent magnetization of PEG-NHs within a few weeks in the acidic medium (Figure 3A). The temperature dependence of the magnetization also indicates some changes in magnetic behavior: the blocking temperature corresponding to maximum magnetization (i.e., the temperature of transition between rigid dipole and superparamagnetic behavior) is shifted toward lower temperatures indicating a decrease of Néel relaxation time, which is consistent with a diminution of the magnetic size over time (Figure 3B). Hence, not only the magnetization of the particles decreases, but also their magnetic dynamics is modified by the transformation of the $\mathrm{NHs}$ in the acidic medium. Likewise, the optical extinction spectrum shows that the absorbance decreases below $500 \mathrm{~nm}$ due to the degradation of iron 


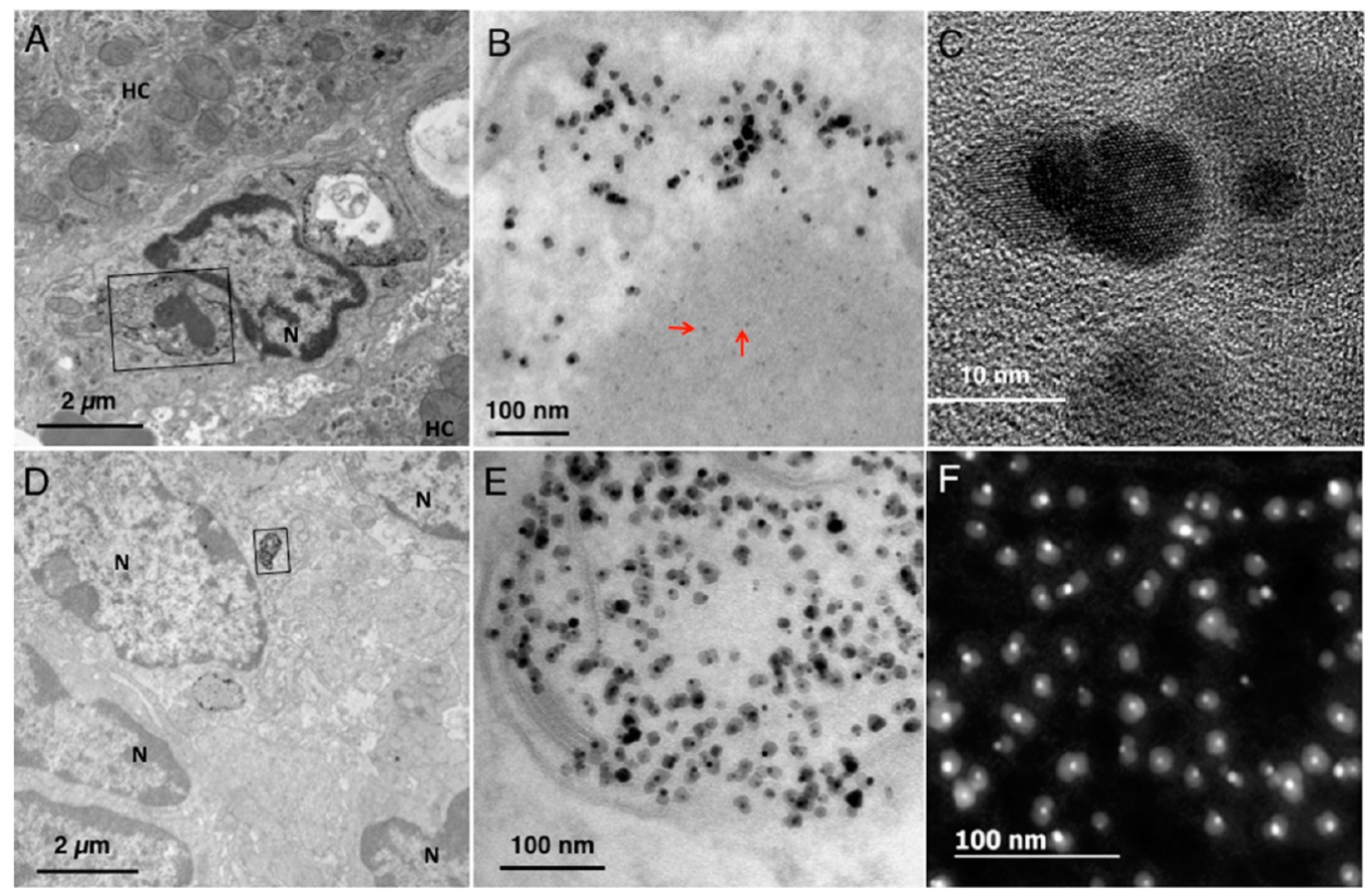

Figure 4. Multiscale TEM tracking of polymer-coated heterostructures in spleen and liver at day 1 postinjection. NHs are confined within intracellular lysosomes of macrophages in liver and spleen. (A) Liver macrophage (= Kupffer cell, $\mathrm{N}$ denotes its nucleus) between two hepatocytes ( $\mathrm{HC}$ ). The square indicates the NH-containing lysosome. (B) Higher magnification of electron dense $\mathrm{NHs}$ coexisting with less dense iron-rich ferritin proteins (red arrows). (C) High-resolution TEM reveals the intact hybrid structure of particles with highly electron dense gold core and medium electron dense iron oxide petals. (D) Splenic macrophages ( $\mathrm{N}$, nucleus; black square, NH-loaded lysosome). (E) Magnified view of the square on (D). (F) High angle annular dark field (HAADF) scanning transmission electron microscopy (STEM) micrograph of intralysosomal $\mathrm{NHs}$ showing the bright gold particles colocalizing with less intense iron oxide nanocrystals.

oxide nanocrystals, making the plasmon resonance peak of the $5 \mathrm{~nm}$ gold particles more apparent (Figure 3C). Hence, the evolution of magnetic and optical properties is in agreement with the observed dissolution of iron oxide around the resilient gold nanocores. It must be noted that the kinetics of dissolution is much faster on TEM grids than in solution, as already reported for nanocubes. ${ }^{32}$ In line with these results, the dissolution of iron oxide nanospheres was shown to depend on the ratio of citrate ions per particle in suspension. ${ }^{16}$

In Vivo Transformation. Prior administrations to animals the particles were dispersed ex tempore in physiological saline. The suspension was well dispersed and was easily administered through an insulin syringe. The administration to the animals of each group took less than $10 \mathrm{~min}$, when both, PEG-coated and PCcoated NHs were equally stable. Mice were intravenously injected with the nominal dose of iron oxide used in clinical practice and preclinical assays for MRI studies ( $50 \mu \mathrm{mol}$ of iron $/ \mathrm{kg}$ ). For a $20 \mathrm{~g}$ weighed mouse, the injected dose is $56 \mu \mathrm{g}$ of iron and $14 \mu \mathrm{g}$ of gold for PC-NHs (12.7 $\mu$ g of gold for the PEG-NHs). Notably, gold administration associated with $\mathrm{NHs}$ is low in comparison to previous studies investigating the distribution of gold nanoparticles. ${ }^{23}$ However, despite the low dose of gold, we were able to track nanoscopic transformations of nanoparticles within the principal organs of accumulation, namely, the spleen and the liver, up to one year after particles administration. Regardless of the time points after injection and the nature of particle coating, the particles were always found within intracellular vesicles, presumably lysosomes, into macrophage-like cells (Kupffer cells in liver and macrophages or Ito cells in spleen) adjacent to hepatocytes (easily recognizable by their regularly circular nucleus and abundant mitochondria and glycogen) (see Supporting Information Figure S13). At day 1 after administration (Figure 4), the hybrid structure of the particles was intact as observed on bright field TEM and high angle annular dark field (HAADF) scanning transmission electron microscopy (STEM) images. Atomic scale resolution can be achieved on single $\mathrm{NHs}$ in organs by imaging ultrafine slices of $30 \mathrm{~nm}$ in thickness. The intact NHs with both PEG-coating (Supporting Information Figure S13) and PC-coating (Figures 4 and 5A) were well dispersed in the lysosomes despite their high local concentration. In addition, the iron-rich ferritin proteins coexisted in proximity to $\mathrm{NHs}$ (red arrows in Figure 4 and Supporting Information Figure S13).

From day 7 and up to 12 months after injection of PC-coated NHs, we still observed intact heterostructures, but also highly electron dense small particles, often forming strings or chains (Figure $5 B, C$ and Supporting Information Figure S14). By analyzing these structures with HRTEM and STEM-EDX nanoanalysis, we identified gold particles with residual iron oxide 


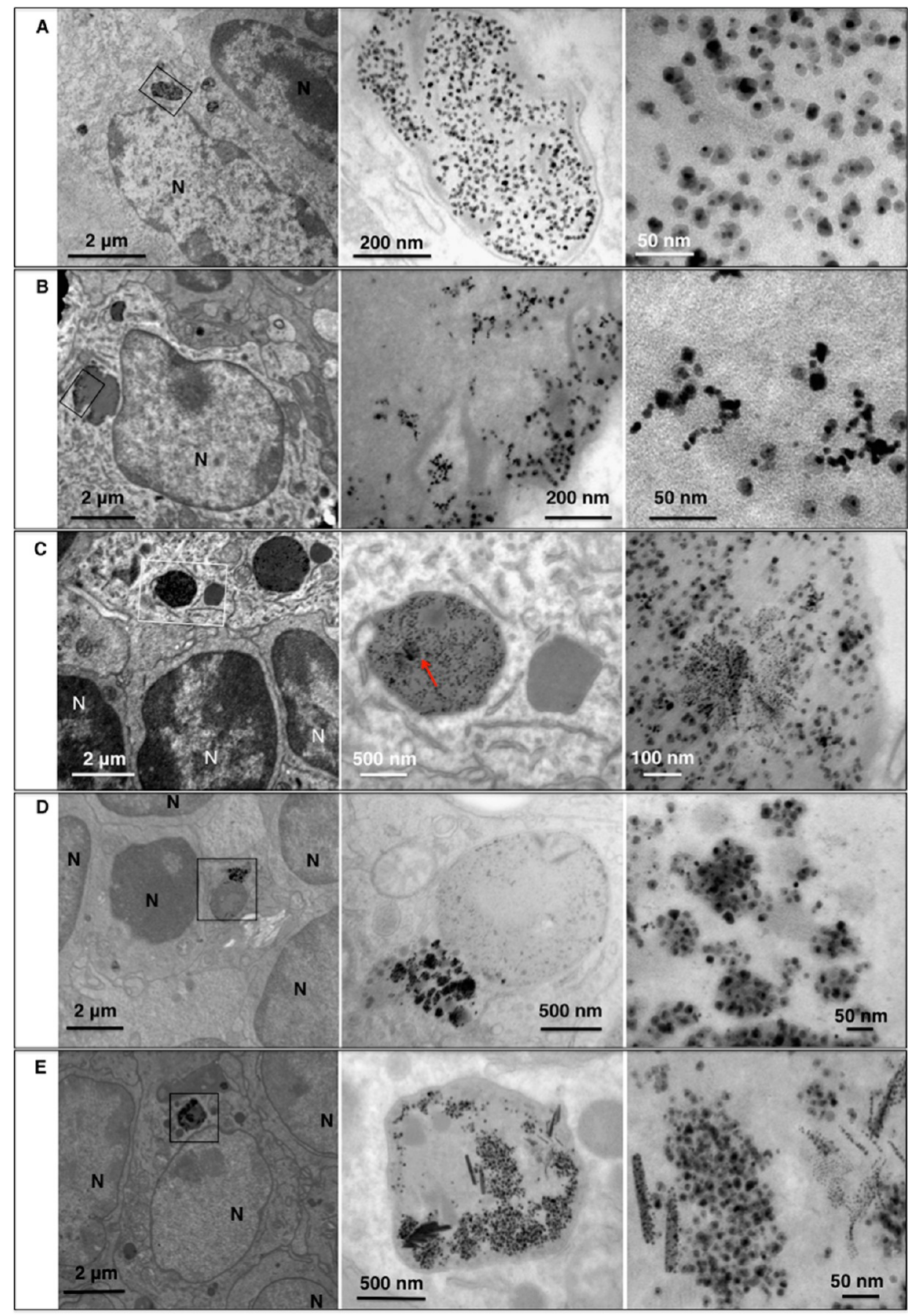

Figure 5. Ultrastructural data of the spleen: (A) Spleen section at D1 when NHs, localized in splenic lysosomes, do not exhibit any particular alterations (with gradual magnifications of the zone in the square). (B) At D7, gold core residues start forming characteristic chains, which coexist with unaltered heterostructures. (C) At D14, some particles start forming complex assemblies made of smaller gold residues (red arrow), zone magnified on the right side. (D) Spleen at D30 and (E) Spleen at D90 where distinctive chains and assemblies of gold residues are manifested together with unaltered heterostructures.

(Figure 6). The presence of iron oxide traces onto gold particles unambiguously confirms iron oxide local dissolution in vivo (Supporting Information Figure S15). Remarkably, the remnant gold particles play the role of long-lived tracers to highlight the local (and sometimes complete) degradation of iron oxide nanocrystals. Resilient gold particles become more frequent over time, both in liver and in spleen, and they surprisingly assemble into longer chains, lattices or clusters (Figure 5B-E day 7 to day 90 in spleen, Figure $7 \mathrm{~A}-\mathrm{C}$ day 90 in spleen, Supporting Information Figures S16 day 90 in liver, Supporting Information Figure S17 day 360 in spleen). Even more compelling, 30 days postinjection the diameters of gold cores diminished to $2-3 \mathrm{~nm}$, in comparison to their initial $5 \mathrm{~nm}$ diameter in heterostructures. Eroded seeds were unambiguously 

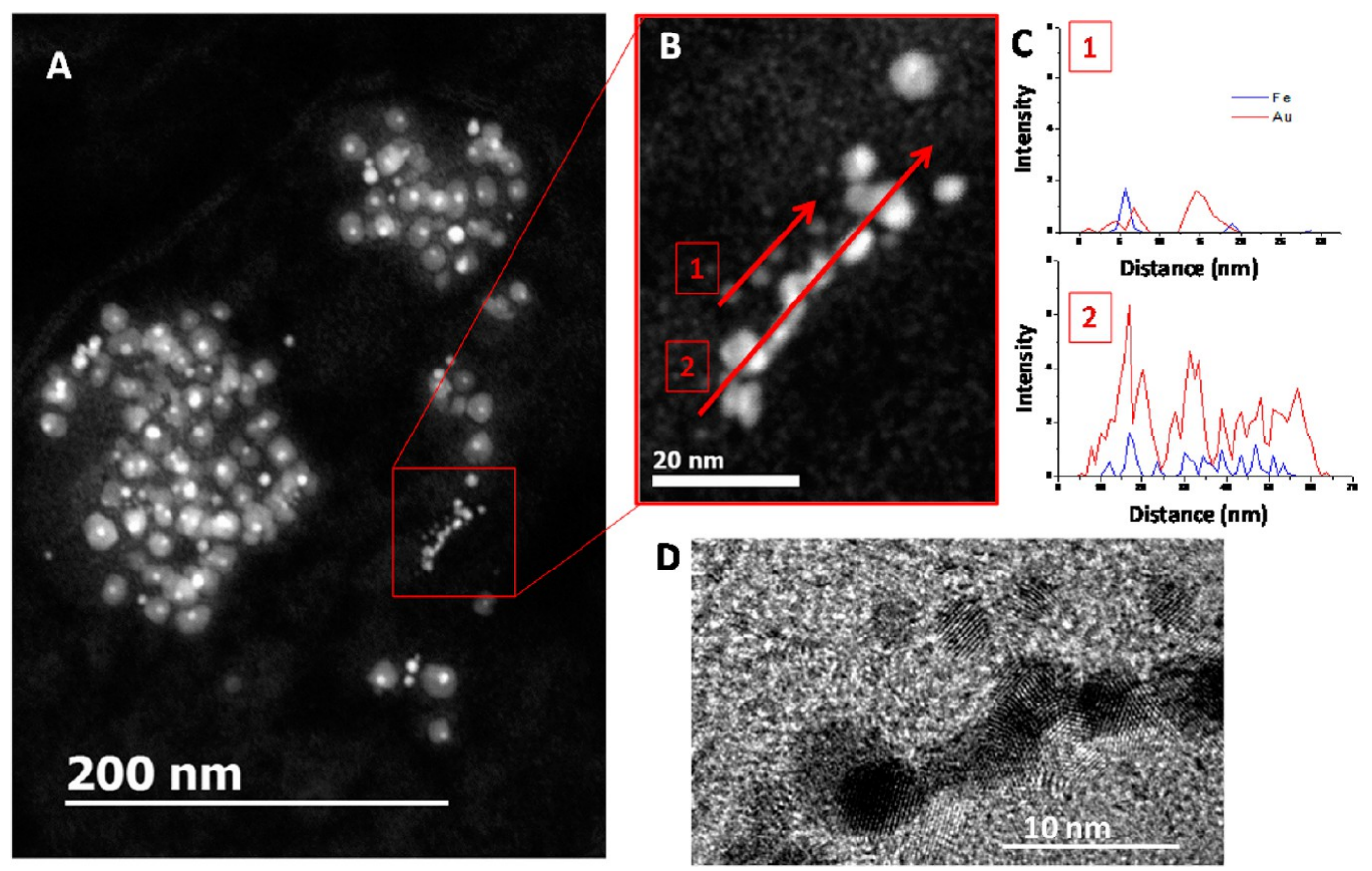

Figure 6. Two-stage element-specific biodegradation of NHs: first stage, dissolution of iron oxide around resilient gold core observed on D14 in spleen. (A) Dark field (STEM-HAADF) micrograph of a splenic lysosome reveals intact NHs close to chainforming bright residues (red square). (B) Magnification of the red square in (A). (C) STEM-EDX nanoanalysis on line 1 and 2 in (B). Iron and gold intensity profiles in line 1 and line 2 reveal partial or total disappearance of iron oxide surrounding the gold core. (D) High-resolution TEM also confirms that some gold particles still have interfaces with iron oxide while others consist of pure gold monocrystals.

identified as gold particles by STEM-EDX (Figure 7B-E). The finding that gold particles undergo progressive degradation inflicted by the local environment is very significant, as the toxicity/translocation/excretion profiles of gold nanoparticles have been related to their size. ${ }^{23}$ It is worth noticing that macrophages are known to produce hydroxyl radicals, which are able to etch noble metal nanoparticles. ${ }^{47}$

We postulate that the biotransformation of $\mathrm{NHs}$ occurs in two stages: first stage is the dissolution of iron oxide crystals that takes place around persistent gold particles (Figure 6) and second stage is the degradation of gold particles to form progressively smaller particles (Figure 7) that tend to self-assemble.

Importantly, the lysosome medium induces uneven dissolution of particles and some $\mathrm{NHs}$ resist to degradation over one year after particle administration (Supporting Information Figure S17). The finding is in line with the step-by-step observation of $\mathrm{NH}$ in acidic citrate medium showing that each heterostructure has its own kinetics of degradation. Similarly, iron oxide nanocubes could display intact shapes following few months after administration to mice, while most cubes have been degraded and lost their magnetic properties. ${ }^{32}$ What protects some particles from dissolution and conversely triggers the degradation of others in vivo is still unclear. We previously reported that the dissolution of iron oxide nanocubes started at locations where the polymer coating was missing or was less dense (typically at cube edges). ${ }^{32}$ The situation in vivo is more complex since NHs interact first with plasma proteins ${ }^{52,53}$ and then with cellular and lysosomal constituents during cell uptake and processing. ${ }^{48,49}$ How does the biological corona influence the nanocrystal degradation within the proteolytic environment of lysosomes is currently unknown. The agglomeration state and local density of nanoparticles in lysosomes also evolve over time: typically, $\mathrm{NHs}$ are confined at relatively high density at short times after injection, whereas they tend to individualize on the lysosome margins or to segregate into some electron dense areas of the lysosomes at longer time-points (see for instance Supporting Information Figures S13 and S16). Cellular metabolism for detoxification of endogenous and xenobiotic hydrophobic compounds may be involved in the redistribution and degradation of the coated heterostructures and of the gold remnants. The presence (or de novo synthesis) of ferritins, the iron storage proteins that are observed in proximity of nanoparticles, suggests their involvement in the degradation mechanisms by incorporating free iron ions that are released from iron oxide crystals.

Another striking point is the spontaneous formation of chains and the assembly of resilient and degraded gold particles. While most $\mathrm{NHs}$ are well separated on day one postinjection due to their polymer shell and/or protein corona, they tend to form chaplets at longer time-points, suggesting that the polymer/ protein shell has been degraded or has detached in lysosomes. The small residual gold particles, which 

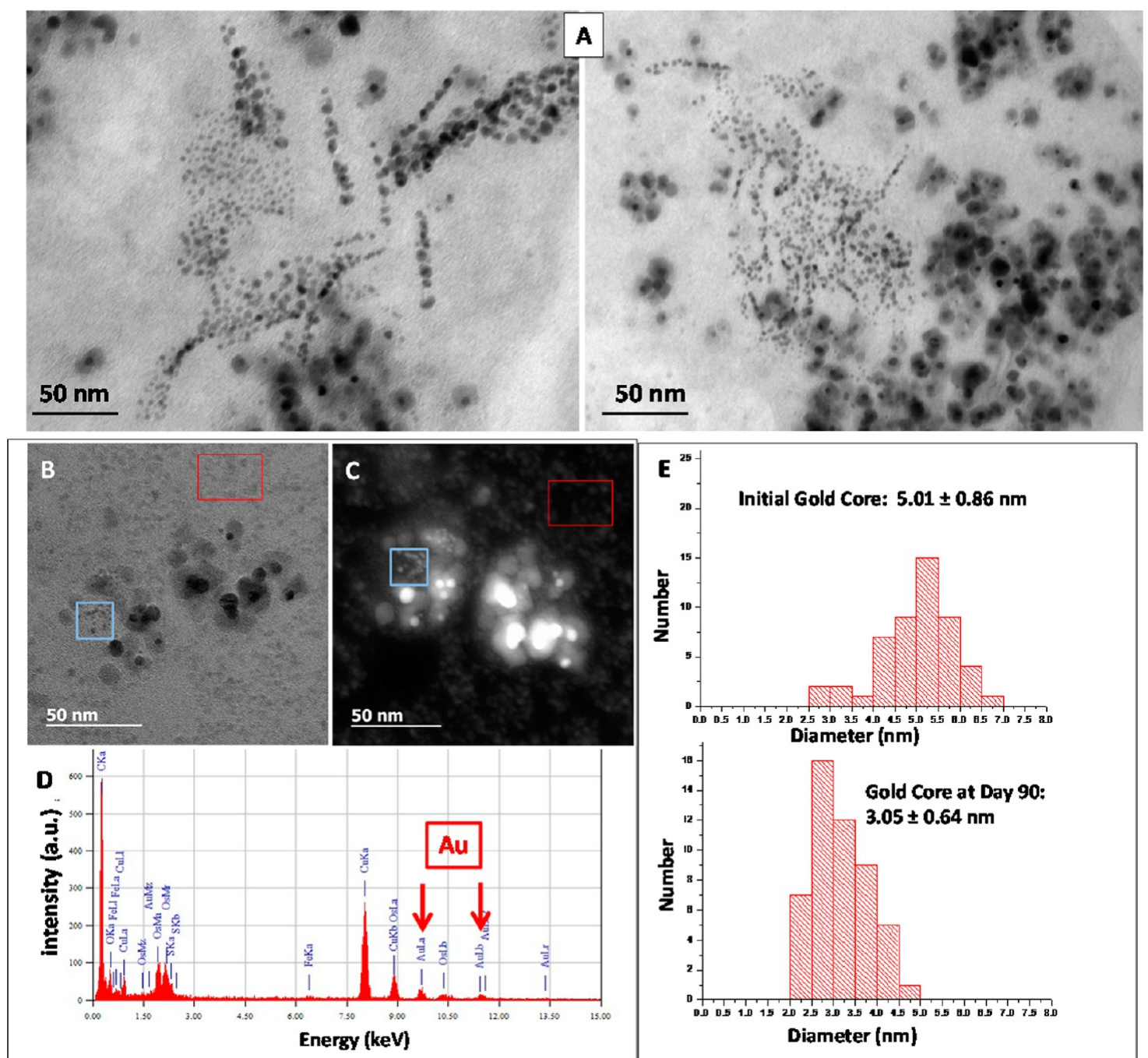

Figure 7. Two-stage element-specific biodegradation of NHs: second stage, degradation of gold particles and formation of gold residues assemblies observed on D90 in spleen. (A and B) Bright field TEM micrographs showing residual intact NHs and degraded NHs assembling together. (C) STEM-HAADF micrograph of (B) showing different contrast of gold residues (blue window) and iron-rich ferritin proteins (red window). (D) STEM-EDX nanoanalysis of the blue window in (C) identifies the electron-dense residues as gold particles characterized by AuLa and AuLb peaks. (E) The size distribution of initial gold cores (mean diameter of $5.01 \pm 0.86 \mathrm{~nm}$ at day 1 in vivo) is shifted to lower sizes (mean diameter of $3.05 \pm 0.64 \mathrm{~nm}$ ) after dissolution of iron oxide at day 90 , revealing the gradual erosion of gold particles.

ensued from the degradation of $5 \mathrm{~nm}$ gold cores also tend to form dynamic superstructures as exemplified in Figure 7. Importantly, neither chain formation nor gold particles degradation and assembly were observed in vitro in acidic citrate medium. This suggests that biological effectors of the lysosomes (responsible for enzymatic and redox activities, for instance) are involved in nanoparticles processing, including individual transformations as well as recurrent reorganization. Of course, further work is needed to decipher the nanobiointeractions occurring in lysosomes over the time of nanoparticles persistence within the body. Interestingly, in a recent study, gold particles were organized with DNA to form superstructures that degrade in macrophages to escape from the body by renal clearance. ${ }^{50}$ Thus far, our study provides direct in vivo evidence of iron oxide dissolution and transformations of gold particles.
Evidence for Elimination of NHs and Role of Surface Coating. The two constitutive materials of heterostructures, iron oxide and gold, were monitored simultaneously up to one year postinjection in liver, spleen, kidney, and lung by inductively coupled plasma-mass spectrometry (ICP-MS) and ESR, respectively (Figure 8). While ICP elemental analysis remains the "gold standard" for the quantification of gold in tissues, iron quantification by elemental analysis is inaccurate as the endogenous iron exceeds the quantities of iron administered within nanoparticles $(56 \mu \mathrm{g})$. In contrast, ESR specifically measures superparamagnetic nanoparticles (with a detection limit of $10 \mathrm{ng}$ of iron) but not diamagnetic or paramagnetic iron due to endogenous iron species or nanoparticles byproducts. ${ }^{17,51}$ Hence, the evolution of ESR signal in organs directly reflects the loss of superparamagnetic iron due to degradation or clearance of $\mathrm{NHs}$. 

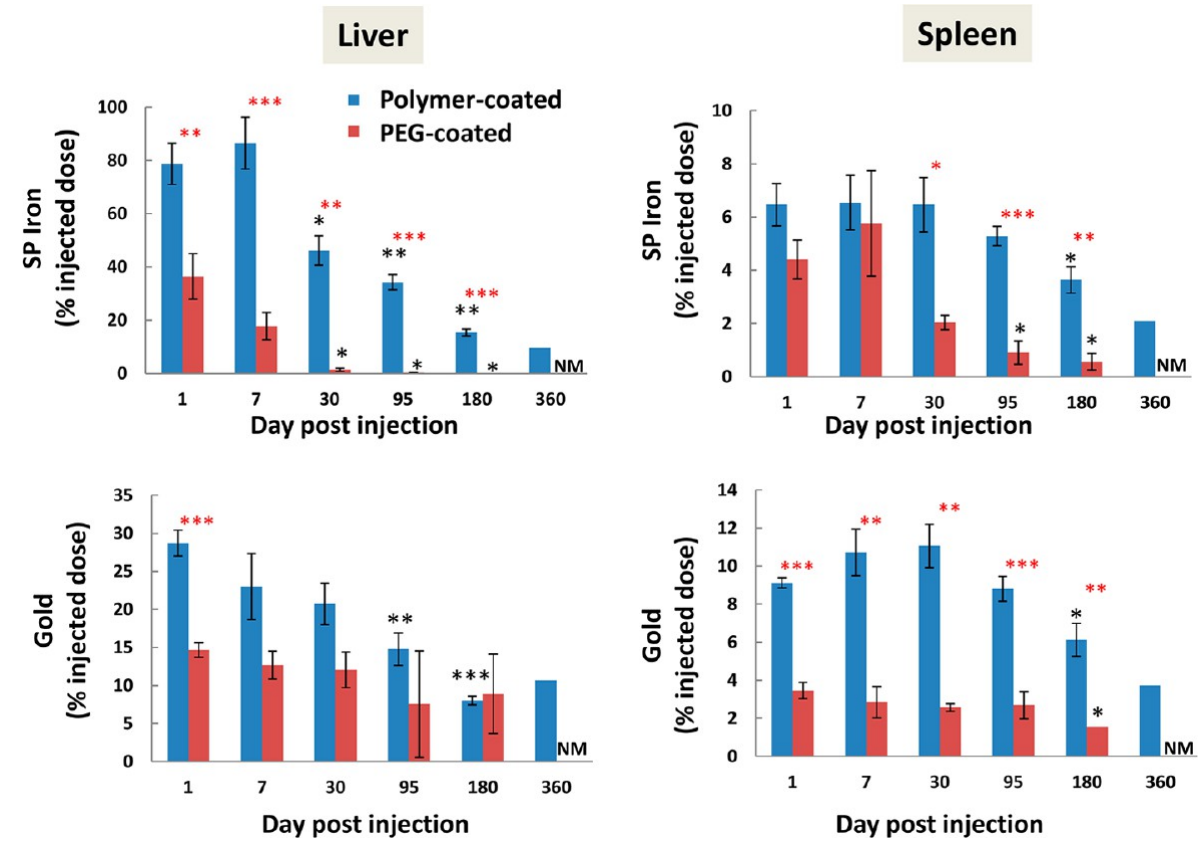

Figure 8. Quantification of superparamagnetic iron (SP iron) measured by ESR and of gold measured by ICP-MS in spleen and liver as a function of time after single administration. SP iron and gold content are expressed as percentage of the injected dose. Bars are standard error of the mean for 4 to 5 mice at each time-point (except at day 360, only 1 mouse for PC-NHs, nonmeasured (NM) for PEG-NHs) (*: $P<0.05,{ }^{* *}: P<0.01,{ }^{* * *}: P<0.001$, black stars are related to Student $t$ test comparing each time-point to D1 for the same coating and red stars comparing PEG-coated and polymer-coated NHs for each time-point).

As expected from previous studies on monocomposite gold or iron oxide nanoparticles and from TEM observations, ${ }^{17,23,31}$ the major uptake of NHs occurred in liver and spleen. The gold content in kidneys and lungs in treated animals was comparable to control animals, irrespective of the time points after injection. Superparamagnetic iron was not detected in lungs, confirming the good dispersion of the injected $\mathrm{NHs}$, which were not blocked in lung capillaries at short times post injection. Magnetic iron oxide was detected in kidneys at a very low dose $(<0.04 \%$ injected dose) and slightly decreased with time (Supporting Information Figure S18), which is consistent with a transient and continuous passage through the kidneys, prior the excretion into the urine. We never observed any pathological histological alterations in these organs (Supporting Information Figure S19). Interestingly, the accumulation in liver increased 2-fold when polymer coating was used in comparison to PEG attaining more than $80 \%$ of the injected dose of iron oxide at day 1 postadministration (42 versus $19 \mu \mathrm{g} / \mathrm{g}$ of magnetic iron in liver for PC-NHs and PEG-NHs, respectively, 51 versus $43 \mu \mathrm{g} / \mathrm{g}$ in spleen) (Figure 8). This is consistent with the camouflaging role of PEG to decrease opsonization and increase the circulation time of nanoparticles. ${ }^{52-54}$ In contrast, the negatively charged amphiphilic polymer is not as efficient to avoid macrophage uptake in liver and spleen.

When looking at the evolution of the quantities of magnetic iron oxide over time, we observe a progressive decrease in concentration in the liver and spleen, which is faster for PEG-NHs in comparison to PC-NHs, and slower in spleen than in liver. Worthy of note is the almost total disappearance of magnetic iron from liver when PEG coating is used. In contrast, one year postinjection, more than $10 \%$ of the injected dose of PC-coated iron oxide still persists in the liver and $2 \%$ in the spleen (corresponding to one-third of the particles concentration at day 1 , i.e., $12.4 \mu \mathrm{g} / \mathrm{g}$ of magnetic iron). Since degradation of iron oxide was unambiguously observed in the spleen and the liver thanks to the gold tracers, we can confirm that the elimination of magnetic iron from these organs is at least partially due to local transformation and dissolution, rather than the excretion of intact particles from the organ. This is important since the organ (and especially the macrophages) must locally process the nanoparticles and manage free iron ions that are released from the particles. One hypothesis is the local transfer of released iron into proximal ferritin proteins, as suggested by TEM observations.

Strikingly, the longer persistence of PC-coated NHs in comparison to PEG-coated counterparts might be the results of the lower degradability rate, as first observed in the acidic citrate medium, suggesting a long-lasting role of the polymer-shell (and associated protein corona) in the protection of the iron oxide crystals in vivo. In contrast, PEG coating offers a more vulnerable shell that may speed up the in situ crystal degradation. The coating also affects the initial NHs concentration in the liver and the spleen, which may in turn influence nanoparticle degradation rate. As we 
observed previously for iron oxide NPs injected at different doses, ${ }^{17}$ the liver may have a limited capacity to handle iron oxide degradation thus longer timeframes are required to manage larger fractions of sequestered nanoparticles. The local overload of iron oxide in macrophages may induce a saturation of the homeostatic capacity of the iron-regulating system.

In line with previous studies investigating the fate of gold particles, ${ }^{23}$ gold originating from $\mathrm{NHs}$ mainly accumulated in liver and spleen (4.9 versus $1.6 \mu \mathrm{g} / \mathrm{g}$ of gold in liver for PC-NHs and PEG-NHs, respectively, 22 versus $10 \mu \mathrm{g} / \mathrm{g}$ in spleen at day 1) (Figure 8). Consistent with the proportions of iron oxide, gold uptake in these organs was about two times larger when PC$\mathrm{NH}$ s were injected in comparison to PEG-NHs, confirming the impact of primary particle shell. ${ }^{53}$ However, by comparing the ratio of magnetic iron over gold content (reported to their respective injected dose) (Supporting Information Figure S20), we observe an excess of magnetic iron in the liver at day 1 and reversely an excess of gold in the spleen. This suggests that a fraction of $\mathrm{NHs}$ is able to fragment to individual gold or iron oxide subunits at early time-points (occurring prior to day 1 after injection) and that the separated subunits have distinct distribution paths due to different sizes or composition. Interestingly, such elementspecific biodistribution has been observed recently for gold-silver nanoshells. ${ }^{55}$

Considering the clearance kinetics, the gold concentration in liver and spleen decreases only slightly in comparison to magnetic iron oxide, the latter decreasing by a factor of 2-fold one year following the administration. This illustrates the long-term persistence of gold nanoseeds, and this finding is in line with previous investigations showing slow and size dependent elimination of PEG-coated gold particles from these organs. ${ }^{56}$ The kinetics of gold retention coming from $\mathrm{NHs}$ is comparable with a previous report on $4 \mathrm{~nm}$ PEGcoated gold particles which peaks at 7 days postinjection and decreases faster than for larger particles. ${ }^{56}$ Remarkably, our TEM observations of the erosion of the $5 \mathrm{~nm}$ gold cores showing their disintegration into smaller particles can account for their long-term elimination if we assume that very small gold particles or molecular gold can be excreted from the liver through hepatobiliary clearance. It has been shown before that small gold NPs of $10 \mathrm{~nm}$ showed the most widespread organ distribution in comparison to larger particles, ${ }^{25}$ suggesting possible translocation between organs. Semmler-Behnke et al. also reported that the translocation of gold NPs from lungs to secondary target organs was inversely proportional to the NP diameter ${ }^{28,57}$ and that the hepato-biliary clearance from liver at $24 \mathrm{~h}$ postinjection strongly increased for $2.8 \mathrm{~nm}$ size and below. ${ }^{58}$ Here, the intralysosomal degradation of gold cores, once iron oxide has been first dissolved, can facilitate the long-term secondary excretion of ultrasmall gold residues from the liver and, to a lesser extent, from the spleen. Conversely, the formation of large assemblies of gold particles within lysosomes may also explain the one-year persistence of gold in these organs. Hence, we clearly observe a materialdependent elimination process, which is related to the different reactivity of gold and iron oxide to the local environment, and is probably also connected to different biological requirements to degrade/reuse/ eliminate the constituents of these two materials.

\section{CONCLUSION}

This study investigates the one year fate of gold/iron oxide nanoheterostructures after intravenous administration to mice. Gold and iron oxide portions of the NHs reveal distinct distribution and elimination kinetics. Heterostructures are degraded following a two-stage process including the primary dissolution of iron oxide crystal around the resilient gold core and the secondary degradation of $5 \mathrm{~nm}$ gold particles into smaller particles that self-organize into chains or assemblies. In situ degradation of gold particles within hepatic and splenic lysosomes can explain the slow elimination of gold from liver and spleen due to translocation and/or excretion mechanisms, which can be set on by the reduced size of gold crystals. On the contrary, large assemblies of gold particles may account for their long-term persistence up to one year postinjection.

The comparison of two different $\mathrm{NHs}$ coatings, amphiphilic polymer or PEG shell, indicates that the synthetic surface identity has long lasting impact on the history of nanoparticles in the body. Our results on hybrid nanoparticles, combining materials with different reactivities, suggest new directions in the control of the degradation, biodistribution, and clearance of multifunctional nanomedicines.

\section{MATERIALS AND METHODS}

Synthesis, Coating, and Characterization of Gold/Iron 0xide NHs. The synthesis method chosen for the production of heterostructures is based on a previously reported protocol by Sun. ${ }^{35}$ Oleic acid $(1.9 \mathrm{~mL}, 90 \%$ purity), oleylamine ( $2 \mathrm{~mL}, 70 \%), 1,2$-hexadecanediol $(2.58 \mathrm{~g}, 90 \%)$ and 1-octadecene $(20 \mathrm{~mL}, 90 \%)$ were placed in a $100 \mathrm{~mL}$ three-neck flask, heated up at $100{ }^{\circ} \mathrm{C}$, and kept under vacuum for $45 \mathrm{~min}$. The temperature was then raised

at $120^{\circ} \mathrm{C}$ and $300 \mu \mathrm{L}$ of iron pentacarbonyl (99.99\% kept in glovebox) was injected. Three minutes later, a mixture of $50 \mathrm{mg}$ of gold(III) chloride trihydrate ( $99.9 \%$, stored under nitrogen) in $0.5 \mathrm{~mL}$ of oleylamine and $5 \mathrm{~mL}$ of octadecene, was added and the temperature was raised from 120 to $310^{\circ} \mathrm{C}$ in $10 \mathrm{~min}$ and kept at $310^{\circ} \mathrm{C}$ for $90 \mathrm{~min}$. After the reaction pot had cooled down, three washing steps were performed by addition of 2-propanol and centrifugation, followed by redispersion of the precipitated heterodimers in hexane. At the last washing 
step, the sample was dissolved in $15 \mathrm{~mL}$ of chloroform, and $50 \mu \mathrm{L}$ of oleylamine was also added.

Water Transfer by Polymer Coating Procedure. The assynthesized batch of heterodimers was transferred from chloroform to water using a polymer coating protocol wellestablished in our group. ${ }^{39}$ A total of $16 \mathrm{~mL}$ of poly(maleic anhydride-alt-1-octadecene) (137 $\mathrm{mM}$ in chloroform) was added to $60 \mathrm{~mL}$ of nanoparticles solution $(0.2 \mu \mathrm{M}$ in chloroform). This corresponds to approximately 500 monomer units per $\mathrm{nm}^{2}$. The nanoparticle concentration was calculated by measuring the gold by elemental analysis, while the total surface area was approximated to a spherical object having an overall diameter given by the sum of the gold and the iron oxide diameters. The solvent was evaporated slowly using a rotary evaporator (at $400 \mathrm{mbar}$ and $40^{\circ} \mathrm{C}$ for $2 \mathrm{~h}$ ). Then, $3.7 \mathrm{~mL}$ of bis(hexamethylene) triamine (20 $\mathrm{mM}$ in chloroform) was added corresponding to 10 cross-linker molecules per $\mathrm{nm}^{2}$. The solution was sonicated for $10 \mathrm{~min}$ at $40{ }^{\circ} \mathrm{C}$. Subsequently, the solvent was removed under reduced pressure (a first step at $400 \mathrm{mbar}$ and $40{ }^{\circ} \mathrm{C}$ for $2 \mathrm{~h}$, then a second step at $200 \mathrm{mbar}$ at $40^{\circ} \mathrm{C}$ for $1 \mathrm{~h}$, and a last step at $100 \mathrm{mbar}$ and $40^{\circ} \mathrm{C}$ for $30 \mathrm{~min}$ until all the solvent is evaporated). Five milliliters of aqueous sodium borate buffer $(50 \mathrm{mM}, \mathrm{pH} 9)$ was added, and the mixture was sonicated at $65^{\circ} \mathrm{C}$ for $2 \mathrm{~h}$. The solution was stored overnight at $65^{\circ} \mathrm{C}$ under shaking, and later concentrated to a volume of only $1-2 \mathrm{~mL}$ by centrifugation filtration with a centricon tube $(15 \mathrm{~mL}$, MWCO $100 \mathrm{kDa}$ ) at about $1700 \mathrm{RCF}$. The polymer coated nanoparticles were cleaned from the excess of polymer by ultracentifugation performed by depositing $1 \mathrm{~mL}$ of sample on a discontinuous sucrose gradient ( $2 \mathrm{~mL} / 10 \%, 3 \mathrm{~mL} / 40 \%, 4 \mathrm{~mL} / 60 \%$ ) and running the centrifugation at about $15000 \mathrm{RCF}$ for $1 \mathrm{~h}$. When using these conditions, the heterodimers sample was placed in the middle part of the gradient while the excess of polymer was stuck on the top of the gradient. The sucrose gradient containing the heterodimers was recovered from the gradient, concentrated, and cleaned from the sucrose on centricone tubes.

Etching Procedure for the Fabrication of Empty Heterostructures. To prepare empty NHs, we have selectively etched the gold nanocrystals from the polymer-coated gold/iron oxide heterostructures, by adjusting a protocol previously developed by us to obtain empty heterostructure directly in organic solution. ${ }^{45}$ To $4 \mathrm{~mL}$ of polymer coated gold/iron oxide $\mathrm{NHs}$ $(0.2 \mu \mathrm{M}$ in water) was added $8 \mathrm{~mL}$ of iodine solution (Tincture of iodine $7 \%$ in $\mathrm{p} / \mathrm{v}$ of iodine and $5 \%$ of potassium iodide in water and ethanol). After a $24 \mathrm{~h}$ reaction at $50^{\circ} \mathrm{C}$ under vigorously shaking, the sample was centrifuged on centrifuge filters (MW $100 \mathrm{kDa}$ ) to remove the excess of iodine compounds and gold ions. Under these conditions, together with the hollow $\mathrm{NHs}$ also a fraction of the initial gold/iron oxide heterostructures was still present. To separate the hollow $\mathrm{NHs}$ fraction from the full one, a purification was performed on a discontinuous sucrose gradient $(4 \mathrm{~mL}$ of $50 \%$ sucrose, $3 \mathrm{~mL}$ of $50 \%$ sucrose solution and $1 \mathrm{~mL}$ of $5 \%$ sucrose solution and a loading sample volume of $0.5 \mathrm{~mL}$ ) at low centrifugation speed and at $4^{\circ}$ (4000 rpm, for $210 \mathrm{~min}$ ). This allowed isolating the hollow $\mathrm{NHs}$ in the middle of the gradient from the full $\mathrm{NHs}$ precipitated at the bottom of the tube. The recovered sample was finally washed by centrifugation to remove the sucrose.

Water Transfer by PEG Ligand Exchange Procedure. Alternatively, to transfer the surfactant coated gold/iron oxide NHs into water, a ligand exchange procedure was employed by choosing the gallic functionalized polyethylene glycol (Gallol-PEG-OH) as the ligand exchange molecules. ${ }^{41}$ To $10 \mathrm{~mL}$ of the ligand Gallol-PEG-OH solution $\left(0.05 \mathrm{M}\right.$ in $\left.\mathrm{CHCl}_{3}\right)$ and $1 \mathrm{~mL}$ of triethylamine was added $5 \mathrm{~mL}$ of stock solution of $\mathrm{NHs}$ such that the final $\mathrm{NH}$ concentration was set at $1 \mathrm{~g} / \mathrm{L}$ of Fe in the $\mathrm{CHCl}_{3}$ phase. The mixture was then diluted with $50 \mathrm{~mL}$ of toluene, shaken, and transferred in a separating funnel. Then, $250 \mathrm{~mL}$ of deionized water were added, resulting in a two-phase mixture that was gently shaken. After the ligand-exchange took placed, the heterostructures were laid at the toluene-water interface. After emulsification by means of shaking, the phases were allowed to separate and the aqueous phase containing $\mathrm{NH}$ s bearing gallolPEG-OH was collected. This step was repeated until all $\mathrm{NHs}$ were transferred into water and the organic phase was completely transparent. The total water volume was brought to
$10 \mathrm{~mL}$ under reduced pressure at $40^{\circ} \mathrm{C}$ (300 mbar for $30 \mathrm{~min}$, $200 \mathrm{mbar}$ for $30 \mathrm{~min}, 77 \mathrm{mbar}$ for $30 \mathrm{~min}$ and $10 \mathrm{mbar}$ for $10 \mathrm{~min}$ ) and the excess of Gallol-PEG-OH was removed by dialysis (membrane molecular cutoff of $50 \mathrm{kDa}$ ) in a deionized water bath. The sample was left in dialysis under stirring for 2 days at room temperature. Finally, the NHs solution was concentrated by centrifugation in a centrifuge filter (molecular cutoff point $100 \mathrm{kDa}$ ), and the recovered solution of PEG-coated $\mathrm{NHs}$ was analyzed by DLS and TEM.

In Vitro Degradation Study. To mimic the lysosomal medium, $\mathrm{NHs}$ were dispersed in a citrate buffer at $\mathrm{pH} 4.7$ at a concentration of $10 \mathrm{mM}$ in iron. The citrate buffer was prepared by mixing $4.4 \mathrm{mM}$ of citric acid $\left(\mathrm{C}_{6} \mathrm{H}_{8} \mathrm{O}_{7}\right.$, Fluka, $\left.>99.5 \%\right)$ and $5.6 \mathrm{mM}$ of sodium citrate tribasic $\left(\mathrm{C}_{6} \mathrm{H}_{5} \mathrm{Na}_{3} \mathrm{O}_{7} \cdot 2 \mathrm{H}_{2} \mathrm{O}\right.$, Fluka, $\left.>99 \%\right)$ in $500 \mathrm{~mL}$ of purified water.

In Vivo Study. The experiments were performed in agreement with institutional animal use and care regulation of the Paris Cardiovascular Research Center animal facility (University Paris Descartes). The animals were allowed to acclimate to this facility for at least 1 week before being used in the experiments and were fed a standard diet ad libitum throughout the experiments. Pathogen-free female 8 week old C57/Bl6 mice (mean weight $20.5 \pm 1 \mathrm{~g}$ ) (Janvier, France)) were injected in the retro-orbital vein with PC-NHs or PEG-NHs suspended in $100 \mu \mathrm{L}$ of physiological saline medium at a dose of $50 \mu \mathrm{mol}$ iron $/ \mathrm{kg}$ (injected dose of $56 \mu \mathrm{g}$ iron). Four mice injected with PC-NHs, four mice injected with PEG-NHs, and five control noninjected mice were sacrificed at each time point after NHs injection (at days D1, D7, D30, D95 and D180, respectively). One mouse injected with PC-NHs was sacrificed at Day 360. Liver, spleen, kidneys, and lungs were excised and prepared for TEM, histology, ESR, and ICP-MS.

Histology. After excision, pieces of liver, spleen, lung, and kidney were fixed with $\mathrm{pH} 7.4$ phosphate-buffered $10 \%$ formalin and processed by embedding in paraffin. Six micrometer thick sections were evaluated after hematoxylin and eosin, Masson's trichrome, or Pearls and Nuclear Fast Red staining.

TEM of Organs. Organs were cut into $1 \mathrm{~mm}^{3}$ pieces after excision and fixed with $2 \%$ glutaraldehyde in $0.1 \mathrm{M}$ sodium cacodylate buffer, postfixed with $1 \%$ osmium tetroxide containing $1.5 \%$ potassium cyanoferrate, gradually dehydrated in ethanol, and embedded in Epon. Thin sections $(70 \mathrm{~nm})$ of selected zones were observed with Zeiss EM902 electron microscope operated at 80 kVe (MIMA2-UR1196Génomique et Physiologie de la Lactation, INRA, Plateau de Microscopie Electronique 78352, Jouy en Josas, France). Ultrathin sections ( $30 \mathrm{~nm}$ ) were sectioned for high-resolution imaging and EDX analysis.

Characterization Techniques. High-Resolution Transmission Electron Microscopy (HRTEM) and Electron Spectroscopy. HRTEM investigations were performed with the JEOL ARM $200 \mathrm{~F}$ microscope operating at $200 \mathrm{kV}$ or $80 \mathrm{kV}$ for ex vivo experiments. This microscope is equipped with a CEOS aberration corrector and a cold field emission gun. The follow-up of degradation of individual NHs by HRTEM was performed on lacey carbon film on which $\mathrm{NHs}$ were deposited, initially observed and then immersed for different times in the lysosome-like medium before recurrent observations. The recognizable patterns of the lacey film facilitate the step-by-step monitoring of the NHs alteration. STEM-EDX nanoanalysis is a powerful technique that couples the imaging and analytical performances of electron microscope. We used this technique in spectrum imaging mode ${ }^{59}$ on ex vivo samples: a STEM image of the area of interest was split into a 64 $\times 64$ array of pixels. The nanometric STEM probe is then positioned over each pixel for a dwell time of $50 \mathrm{~ms}$ to acquire a complete energy dispersive $\mathrm{X}$-ray spectrum (multiple scans were used to improve signal-to-noise). The resulting multidimensional datacube encloses spectral information for each pixel from which quantitative chemical information can be extracted. We also performed single point EDX analyses ${ }^{60}$ with longer acquisition times (up to $60 \mathrm{~s}$ ) to reveal the biodistribution of small gold and iron oxide particles.

Magnetic Measurements. Magnetization measurements were carried out on a vibrating sample magnetometer (PPMS, Quantum Design, Inc.) on colloidal suspension of PC-NHs and PEG-NHs in water and in lysosome-like medium. Field-dependent magnetization curves were measured at $310 \mathrm{~K}$ as a function of the external magnetic field in the range between 0 and $3 \times 10^{4} \mathrm{G}$. 
Temperature-dependent magnetization at a magnetic field of $50 \mathrm{G}$ was recorded in the $5-310 \mathrm{~K}$ temperature range for zerofield-cooled sample.

Electron Spin Resonance (ESR). ESR was performed with a Varian E102 EPR (Electron Paramagnetic Resonance) spectrometer operating at $X$ band $(9.26 \mathrm{GHz})$. The first derivative of the power absorption $\mathrm{d} W(\mathrm{~B}) / \mathrm{dB}$ was recorded as a function of the applied field $B$ in the range $0-6000 \mathrm{G}$. The modulation field had a frequency of $100 \mathrm{kHz}$ and amplitude of $10 \mathrm{G}$. The area of the ESR absorption curve (calculated by a double integration of the spectrum $\mathrm{d} W(\mathrm{~B}) / \mathrm{dB}$ ) was proportional to the amount of nondegraded superparamagnetic NHs in the sample. An absolute calibration was performed using suspensions of initial PC-NHs and PEG-NHs ( $2 \mu \mathrm{L}$ sample) at different iron concentrations quantified by ICP-MS. Quantification of $\mathrm{NHs}$ in lysosome-like medium at different time-points was performed on $2 \mu \mathrm{L}$ liquid sample. Before ESR measurements, the excised pieces of organs were first weighed and let dry for 3 days in an oven at $80^{\circ} \mathrm{C}$. The dried organ samples were weighed again and introduced into a quartz tube. The net mass of dehydrated organs in the quartz tube was then weighed and ESR spectra were recorded.

Inductively Coupled Plasma-Mass Spectrometry (ICP-MS). Total iron and gold content in suspension and organs was quantified by ICP-MS. After excision, organ samples were conserved at $-80^{\circ} \mathrm{C}$ until preparation for ICP-MS measurements. The organs were mineralized in closed vial and in the presence of $4 \mathrm{~mL}$ of nitric acid at $70{ }^{\circ} \mathrm{C}$ for $2 \mathrm{~h}$ on heat bloc (DigiPREP Jr SCP Science) and then heat in open vial at $80^{\circ} \mathrm{C}$ for 10 more hours. The volume of each sample was adjusted to 20 or $4 \mathrm{~mL}$ with $3 \%$ nitric acid in distilled water and analyzed for Fe and Au content by ICP-MS (Thermo Electron). A multielement solution (Tune A) was used to optimized the experimental parameter to obtain the maximum counting rates. Data acquisition was made in peak jump mode with three runs for each sample (20 s long, 100 sweep per run, dwell time $200 \mathrm{~ms}$ ). The ICP-MS set up was calibrated with a freshly prepared serial dilution of gold and iron standard using concentration values between 1 and $100 \mathrm{ppb}$ for gold and from 10 to $1000 \mathrm{ppb}$ for iron. Gold detection limits due to instrumental noise were evaluated from the minimal concentration at which ICP measurement is no more linear with concentration (about 5-10 ppb for gold). Noninjected organs were also measured to evaluate endogenous gold concentrations, which were below detection limits.

Optical Absorbance. Extinction spectra in aqueous suspension of $\mathrm{NHs}$ and in lysosome-like medium were measured on a Cary 50 UV/vis spectrometer (Varian).

Conflict of Interest: The authors declare no competing financial interest.

Supporting Information Available: Further characterizations and methods (characterizations of the particle coatings and stability, degradation of PEG-coated NHs in acidic medium, TEM micrographs of PEG-coated and polymer-coated NHs at different time-points in spleen and liver). The Supporting Information is available free of charge on the ACS Publications website at DOI: $10.1021 / a c s n a n o .5 b 00042$.

Acknowledgment. This work was supported by ANR (Agence Nationale de la Recherche) and CGI (Commissariat à l'Investissement d'Avenir) through Labex SEAM (Science and Engineering for Advanced Materials and devices; ANR 11 LABX 086, ANR 11 IDEX 05 02), by the European project Magnifyco (Contract NMP4SL-2009-228622), by the Region lle de France for convention SESAME E1845 and by the CNRS (Defi Nano) and DIM C'Nano lle de France. We are grateful to C. Wilhelm and F. Mazuel for fruitful discussion, to the personnel of the Paris Cardiovascular Research Center (PARCC) for animal facility, especially to L. Pidial, to F. Gendron, Y. Frappart and S. Lajnef for ESR and magnetic experiments, to C. Longin, S. Chat, M. Letheule at the platform MIMA2 at Inra of Jouy en Josas for TEM preparation and observations.

\section{REFERENCES AND NOTES}

1. Lowry, G. V.; Gregory, K. B.; Apte, S. C.; Lead, J. R. Transformations of Nanomaterials in the Environment. Environ. Sci. Technol. 2012, 46, 6893-6899.
Thomas, C. R.; George, S.; Horst, A. M.; Ji, Z.; Miller, R. J.; Peralta-Videa, J. R.; Xia, T.; Pokhrel, S.; Mädler, L.; GardeaTorresdey, J. L.; et al. Nanomaterials in the Environment: From Materials to High-Throughput Screening to Organisms. ACS Nano 2011, 5, 13-20.

3. Winkler, D. A.; Mombelli, E.; Pietroiusti, A.; Tran, L.; Worth, A.; Fadeel, B.; McCall, M. J. Applying Quantitative Structure-Activity Relationship Approaches to Nanotoxicology: Current Status and Future Potential. Toxicology 2013, 313, 15-23.

4. Lynch, I.; Weiss, C.; Valsami-Jones, E. A Strategy for Grouping of Nanomaterials Based on Key Physico-Chemical Descriptors as a Basis for Safer-by-Design Nms. Nano Today 2014, 9, 266-270.

5. Nyström, A. M.; Fadeel, B. Safety Assessment of Nanomaterials: Implications for Nanomedicine. J. Controlled Release 2012, 161, 403-408.

6. Kunzmann, A.; Andersson, B.; Thurnherr, T.; Krug, H.; Scheynius, A.; Fadeel, B. Toxicology of Engineered Nanomaterials: Focus on Biocompatibility, Biodistribution and Biodegradation. Biochim. Biophys. Acta, Gen. Subj. 2011, 1810, 361-373.

7. Lin, Z.; Monteiro-Riviere, N. A.; Riviere, J. E. Pharmacokinetics of Metallic Nanoparticles. Wiley Interdiscip. Rev.: Nanomed. Nanobiotechnol. 2015, 7, 189-217.

8. Fadeel, B.; Feliu, N.; Vogt, C.; Abdelmonem, A. M.; Parak, W. J. Bridge over Troubled Waters: Understanding the Synthetic and Biological Identities of Engineered Nanomaterials. Wiley Interdiscip. Rev.: Nanomed. Nanobiotechnol. 2013, 5, 111-129.

9. Loeve, S.; Vincent, B. B.; Gazeau, F. Nanomedicine Metaphors: From War to Care. Emergence of an Oecological Approach. Nano Today 2013, 8, 560-565.

10. Chen, N.; Wang, H.; Huang, Q.; Li, J.; Yan, J.; He, D.; Fan, C.; Song, $H$. Long-Term Effects of Nanoparticles on Nutrition and Metabolism. Small 2014, 10, 3603-3611.

11. Kwon, D.; Nho, H. W.; Yoon, T. H. X-Ray and Electron Microscopy Studies on the Biodistribution and Biomodification of Iron Oxide Nanoparticles in Daphnia Magna. Colloids Surf., B 2014, 122, 384-389.

12. Mudunkotuwa, I. A.; Pettibone, J. M.; Grassian, V. H. Environmental Implications of Nanoparticle Aging in the Processing and Fate of Copper-Based Nanomaterials. Environ. Sci. Technol. 2012, 46, 7001-7010.

13. Tenzer, S.; Docter, D.; Kuharev, J.; Musyanovych, A.; Fetz, V.; Hecht, R.; Schlenk, F.; Fischer, D.; Kiouptsi, K.; Reinhardt, C.; et al. Rapid Formation of Plasma Protein Corona Critically Affects Nanoparticle Pathophysiology. Nat. Nanotechnol. 2013, 8, 772-781.

14. Aggarwal, P.; Hall, J. B.; McLeland, C. B.; Dobrovolskaia, M. A.; McNeil, S. E. Nanoparticle Interaction with Plasma Proteins as It Relates to Particle Biodistribution, Biocompatibility and Therapeutic Efficacy. Adv. Drug Delivery Rev. 2009, 61, 428-437.

15. Liu, J.; Wang, Z.; Liu, F. D.; Kane, A. B.; Hurt, R. H. Chemical Transformations of Nanosilver in Biological Environments. ACS Nano 2012, 6, 9887-9899.

16. Levy, M.; Lagarde, F.; Maraloiu, V. A.; Blanchin, M. G.; Gendron, F.; Wilhelm, C.; Gazeau, F. Degradability of Superparamagnetic Nanoparticles in a Model of Intracellular Environment: Follow-up of Magnetic, Structural and Chemical Properties. Nanotechnology 2010, 21, 395103.

17. Levy, M.; Luciani, N.; Alloyeau, D.; Elgrabli, D.; Deveaux, V.; Pechoux, C.; Chat, S.; Wang, G.; Vats, N.; Gendron, F.; et al. Long Term in Vivo Biotransformation of Iron Oxide Nanoparticles. Biomaterials 2011, 32, 3988-3999.

18. Dreaden, E. C.; Alkilany, A. M.; Huang, X.; Murphy, C. J.; El-Sayed, M. A. The Golden Age: Gold Nanoparticles for Biomedicine. Chem. Soc. Rev. 2012, 41, 2740-2779.

19. Yoo, D.; Lee, J.-H.; Shin, T.-H.; Cheon, J. Theranostic Magnetic Nanoparticles. Acc. Chem. Res. 2011, 44, 863-874.

20. O'Neal, D. P.; Hirsch, L. R.; Halas, N. J.; Payne, J. D.; West, J. L. Photo-Thermal Tumor Ablation in Mice Using near Infrared-Absorbing Nanoparticles. Cancer Lett. 2004, 209, $171-176$. 
21. Kolosnjaj-Tabi, J.; Di Corato, R.; Lartigue, L.; Marangon, I.; Guardia, P.; Silva, A. K. A.; Luciani, N.; Clément, O.; Flaud, P.; Singh, J. V.; et al. Heat-Generating Iron Oxide Nanocubes: Subtle "Destructurators" of the Tumoral Microenvironment. ACS Nano 2014, 8, 4268-4283.

22. Jenkins, J. T.; Halaney, D. L.; Sokolov, K. V.; Ma, L. L.; Shipley, H. J.; Mahajan, S.; Louden, C. L.; Asmis, R.; Milner, T. E.; Johnston, K. P.; et al. Excretion and Toxicity of Gold-Iron Nanoparticles. Nanomedicine 2013, 9, 356-365.

23. Khlebtsov, N.; Dykman, L. Biodistribution and Toxicity of Engineered Gold Nanoparticles: A Review of in Vitro and in Vivo Studies. Chem. Soc. Rev. 2011, 40, 1647-1671.

24. Pan, Y.; Neuss, S.; Leifert, A.; Fischler, M.; Wen, F.; Simon, U.; Schmid, G.; Brandau, W.; Jahnen-Dechent, W. SizeDependent Cytotoxicity of Gold Nanoparticles. Small 2007, 3, 1941-1949.

25. De Jong, W. H.; Hagens, W. I.; Krystek, P.; Burger, M. C.; Sips, A. J. A. M.; Geertsma, R. E. Particle Size-Dependent Organ Distribution of Gold Nanoparticles after Intravenous Administration. Biomaterials 2008, 29, 1912-1919.

26. Guo, L.; Panderi, I.; Yan, D. D.; Szulak, K.; Li, Y.; Chen, Y.-T.; Ma, H.; Niesen, D. B.; Seeram, N.; Ahmed, A.; et al. A Comparative Study of Hollow Copper Sulfide Nanoparticles and Hollow Gold Nanospheres on Degradability and Toxicity. ACS Nano 2013, 7, 8780-8793.

27. Brown, C. L.; Whitehouse, M. W.; Tiekink, E. R. T.; Bushell, G. R. Colloidal Metallic Gold Is Not Bio-Inert. Inflammopharmacology 2008, 16, 133-137.

28. Semmler-Behnke, M.; Kreyling, W. G.; Lipka, J.; Fertsch, S.; Wenk, A.; Takenaka, S.; Schmid, G.; Brandau, W. Biodistribution of 1.4- and 18-Nm Gold Particles in Rats. Small 2008, 4, 2108-2111.

29. Sadauskas, E.; Danscher, G.; Stoltenberg, M.; Vogel, U.; Larsen, A.; Wallin, H. Protracted Elimination of Gold Nanoparticles from Mouse Liver. Nanomedicine 2009, 5 , 162-169.

30. Weissleder, R.; Stark, D. D.; Engelstad, B. L.; Bacon, B. R.; Compton, C. C.; White, D. L.; Jacobs, P.; Lewis, J. Superparamagnetic Iron Oxide: Pharmacokinetics and Toxicity. AJR, Am. J. Roentgenol. 1989, 152, 167-173.

31. Bourrinet, P.; Bengele, H. H.; Bonnemain, B.; Dencausse, A.; Idee, J. M.; Jacobs, P. M.; Lewis, J. M. Preclinical Safety and Pharmacokinetic Profile of Ferumoxtran-10, an Ultrasmall Superparamagnetic Iron Oxide Magnetic Resonance Contrast Agent. Invest. Radiol. 2006, 41, 313-324.

32. Lartigue, L.; Alloyeau, D.; Kolosnjaj-Tabi, J.; Javed, Y.; Guardia, P.; Riedinger, A.; Péchoux, C.; Pellegrino, T.; Wilhelm, C.; Gazeau, F. Biodegradation of Iron Oxide Nanocubes: High-Resolution in Situ Monitoring. ACS Nano 2013, 7, 3939-3952.

33. Javed, Y.; Lartigue, L.; Hugounenq, P.; Vuong, Q. L.; Gossuin, Y.; Bazzi, R.; Wilhelm, C.; Ricolleau, C.; Gazeau, F.; Alloyeau, D. Biodegradation Mechanisms of Iron Oxide Monocrystalline Nanoflowers and Tunable Shield Effect of Gold Coating. Small 2014, 10, 3325-3337.

34. Soenen, S. J. H.; Himmelreich, U.; Nuytten, N.; Pisanic, T. R.; Ferrari, A.; De Cuyper, M. Intracellular Nanoparticle Coating Stability Determines Nanoparticle Diagnostics Efficacy and Cell Functionality. Small 2010, 6, 2136-2145.

35. Yu, H.; Chen, M.; Rice, P. M.; Wang, S. X.; White, R. L.; Sun, S. Dumbbell-Like Bifunctional Au-Fe3o4 Nanoparticles. Nano Lett. 2005, 5, 379-382.

36. Kovalenko, M. V.; Bodnarchuk, M. I.; Lechner, R. T.; Hesser, G.; Schäffler, F.; Heiss, W. Fatty Acid Salts as Stabilizers in Size- and Shape-Controlled Nanocrystal Synthesis: The Case of Inverse Spinel Iron Oxide. J. Am. Chem. Soc. 2007, 129, 6352-6353.

37. Wang, Z. L. Transmission Electron Microscopy of ShapeControlled Nanocrystals and Their Assemblies. J. Phys. Chem. B 2000, 104, 1153-1175.

38. Van Oosterhout, G. W.; Rooijmans, C. J. M. A New Superstructure in Gamma-Ferric Oxide. Nature 1958, 181, 44-44.

39. Pellegrino, T.; Manna, L.; Kudera, S.; Liedl, T.; Koktysh, D.; Rogach, A. L.; Keller, S.; Rädler, J.; Natile, G.; Parak, W. J. Hydrophobic Nanocrystals Coated with an Amphiphilic
Polymer Shell: A General Route to Water Soluble Nanocrystals. Nano Lett. 2004, 4, 703-707.

40. Di Corato, R.; Quarta, A.; Piacenza, P.; Ragusa, A.; Figuerola, A.; Buonsanti, R.; Cingolani, R.; Manna, L.; Pellegrino, T. Water Solubilization of Hydrophobic Nanocrystals by Means of Poly(Maleic Anhydride-Alt-1-Octadecene) J. Mater. Chem. 2008, 18, 1991-1996.

41. Guardia, P.; Riedinger, A.; Nitti, S.; Pugliese, G.; Marras, S.; Genovese, A.; Materia, M. E.; Lefevre, C.; Manna, L.; Pellegrino, T. One Pot Synthesis of Monodisperse Water Soluble Iron Oxide Nanocrystals with High Values of the Specific Absorption Rate. J. Mater. Chem. B 2014, 2, 4426-4434.

42. Amstad, E.; Zurcher, S.; Mashaghi, A.; Wong, J. Y.; Textor, M.; Reimhult, E. Surface Functionalization of Single Superparamagnetic Iron Oxide Nanoparticles for Targeted Magnetic Resonance Imaging. Small 2009, 5, 1334-1342.

43. Lartigue, L.; Wilhelm, C.; Servais, J.; Factor, C.; Dencausse, A.; Bacri, J.-C.; Luciani, N.; Gazeau, F. Nanomagnetic Sensing of Blood Plasma Protein Interactions with Iron Oxide Nanoparticles: Impact on Macrophage Uptake. ACS Nano 2012, 6, 2665-2678.

44. Arbab, A. S.; Wilson, L. B.; Ashari, P.; Jordan, E. K.; Lewis, B. K.; Frank, J. A. A Model of Lysosomal Metabolism of Dextran Coated Superparamagnetic Iron Oxide (Spio) Nanoparticles: Implications for Cellular Magnetic Resonance Imaging. NMR Biomed. 2005, 18, 383-389.

45. George, C.; Dorfs, D.; Bertoni, G.; Falqui, A.; Genovese, A.; Pellegrino, T.; Roig, A.; Quarta, A.; Comparelli, R.; Curri, M. L.; et al. A Cast-Mold Approach to Iron Oxide and Pt/Iron Oxide Nanocontainers and Nanoparticles with a Reactive Concave Surface. J. Am. Chem. Soc. 2011, 133, 2205-2217.

46. Gazeau, F.; Shilov, V.; Bacri, J.-C.; Dubois, E.; Gendron, F.; Perzynski, R.; Raikher, Y. L.; Stepanov, V. I. Magnetic Resonance of Nanoparticles in a Ferrofluid: Evidence of Thermofluctuational Effects. J. Magn. Magn. Mater. 1999, 202, 535-546.

47. Chee, S. W.; Pratt, S. H.; Hattar, K.; Duquette, D.; Ross, F. M.; Hull, R. Studying Localized Corrosion Using Liquid Cell Transmission Electron Microscopy. Chem. Commun. 2015, 51, 168-171.

48. Wang, F.; Yu, L.; Monopoli, M. P.; Sandin, P.; Mahon, E.; Salvati, A.; Dawson, K. A. The Biomolecular Corona Is Retained During Nanoparticle Uptake and Protects the Cells from the Damage Induced by Cationic Nanoparticles until Degraded in the Lysosomes. Nanomedicine 2013, 9, 1159-1168.

49. Bertoli, F.; Davies, G.-L.; Monopoli, M. P.; Moloney, M.; Gun'ko, Y. K.; Salvati, A.; Dawson, K. A. Magnetic Nanoparticles to Recover Cellular Organelles and Study the Time Resolved Nanoparticle-Cell Interactome Throughout Uptake. Small 2014, 10, 3307-3315.

50. Chou, L. Y. T.; Zagorovsky, K.; Chan, W. C. W. DNA Assembly of Nanoparticle Superstructures for Controlled Biological Delivery and Elimination. Nat. Nanotechnol. 2014, 9, 148-155.

51. Chertok, B.; Cole, A. J.; David, A. E.; Yang, V. C. Comparison of Electron Spin Resonance Spectroscopy and InductivelyCoupled Plasma Optical Emission Spectroscopy for Biodistribution Analysis of Iron-Oxide Nanoparticles. Mol. Pharmaceutics 2010, 7, 375-385.

52. Knop, K.; Hoogenboom, R.; Fischer, D.; Schubert, U. S. Poly(Ethylene Glycol) in Drug Delivery: Pros and Cons as Well as Potential Alternatives. Angew. Chem., Int. Ed. 2010, 49, 6288-6308.

53. Lipka, J.; Semmler-Behnke, M.; Sperling, R. A.; Wenk, A.; Takenaka, S.; Schleh, C.; Kissel, T.; Parak, W. J.; Kreyling, W. G. Biodistribution of Peg-Modified Gold Nanoparticles Following Intratracheal Instillation and Intravenous Injection. Biomaterials 2010, 31, 6574-6581.

54. Cole, A. J.; David, A. E.; Wang, J.; Galbán, C. J.; Yang, V. C. Magnetic Brain Tumor Targeting and Biodistribution of Long-Circulating Peg-Modified, Cross-Linked StarchCoated Iron Oxide Nanoparticles. Biomaterials 2011, 32, 6291-6301. 
55. Goodman, A. M.; Cao, Y.; Urban, C.; Neumann, O.; AyalaOrozco, C.; Knight, M. W.; Joshi, A.; Nordlander, P.; Halas, N. J. The Surprising in Vivo Instability of near-Ir-Absorbing Hollow Au-Ag Nanoshells. ACS Nano 2014, 8, 3222-3231.

56. Cho, W.-S.; Cho, M.; Jeong, J.; Choi, M.; Han, B. S.; Shin, H.-S.; Hong, J.; Chung, B. H.; Jeong, J.; Cho, M.-H. Size-Dependent Tissue Kinetics of Peg-Coated Gold Nanoparticles. Toxicol. Appl. Pharmacol. 2010, 245, 116-123.

57. Kreyling, W. G.; Hirn, S.; Möller, W.; Schleh, C.; Wenk, A.; Celik, G.; Lipka, J.; Schäffler, M.; Haberl, N.; Johnston, B. D.; et al. Air-Blood Barrier Translocation of Tracheally Instilled Gold Nanoparticles Inversely Depends on Particle Size. ACS Nano 2014, 8, 222-233.

58. Hirn, S.; Semmler-Behnke, M.; Schleh, C.; Wenk, A.; Lipka, J.; Schäffler, M.; Takenaka, S.; Möller, W.; Schmid, G.; Simon, U.; et al. Particle Size-Dependent and Surface ChargeDependent Biodistribution of Gold Nanoparticles after Intravenous Administration. Eur. J. Pharm. Biopharm. 2011, 77, 407-416.

59. Herzing, A. A.; Watanabe, M.; Edwards, J. K.; Conte, M.; Tang, Z.-R.; Hutchings, G. J.; Kiely, C. J. Energy Dispersive X-Ray Spectroscopy of Bimetallic Nanoparticles in an Aberration Corrected Scanning Transmission Electron Microscope. Faraday Discuss. 2008, 138, 337-351.

60. Alloyeau, D.; Prévot, G.; Le Bouar, Y.; Oikawa, T.; Langlois, C.; Loiseau, A.; Ricolleau, C. Ostwald Ripening in Nanoalloys: When Thermodynamics Drives a Size-Dependent Particle Composition. Phys. Rev. Lett. 2010, 105, 255901. 